\title{
PROPAGATION OF ANALYTICITY FOR SOLUTIONS OF DIFFERENTIAL EQUATIONS OF PRINCIPAL TYPE
}

\author{
BY KARL GUSTAV ANDERSSON
}

Communicated by P. D. Lax, October 18, 1971

Let $P=P(x, D)$ be a linear differential operator of order $m$ with analytic coefficients in the open set $X \subset R^{n}$, such that

$$
d_{\xi} P_{m}(x, \xi) \neq 0, \quad \text { when }(x, \xi) \in Z=\left\{(x, \xi) \in X \times R^{\eta} \backslash\{0\} ; P_{m}(x, \xi)=0\right\} \text {. }
$$

We shall also assume that $P$ satisfies one of the following three conditions :

(a) The principal part $P_{m}(x, D)$ of $P$ is real.

(b) $d_{\xi} \operatorname{Re} P_{m}$ and $d_{\xi} \operatorname{Im} P_{m}$ are linearly independent in $Z$ and the Poisson bracket $\left\{\operatorname{Re} P_{m}, \operatorname{Im} P_{m}\right\}$ vanishes there.

(c) $P=P(D)$ has constant coefficients.

THEOREM 1. Suppose that $P$ satisfies either (a), (b) or (c). Then the analytic wave front set (see [4]) of a distribution $u$, such that Pu is analytic, is a union of entire bicharacteristic strips.

REMARK 1. In the case (b) the bicharacteristic strips are two-dimensional submanifolds of $Z$ generated by the Hamilton fields $H_{\mathrm{Re} \boldsymbol{P}_{m}}$ and $H_{\mathrm{Im} \boldsymbol{P}_{m}}$ and for operators satisfying (c) they are linear manifolds of the form $\left\{\left(x+d_{\xi} \operatorname{Re}\left(\alpha P_{m}(\xi)\right), \xi\right) ; \alpha \in C\right\}$ for some $x$ and some $\xi$ with $P_{m}(\xi)=0$. Here their dimension may vary between 1 and 2 .

REMARK 2. For the local version, in $X \times R^{\eta} \backslash\{0\}$, of the theorem we need of course only to make the assumptions on $P$ locally.

REMARK 3. When $P$ satisfies (a) and (c) the corresponding regularity theorem in $X$ was proved in [1]. For arbitrary operators satisfying (a) the more precise result concerning analytic wave front sets has been proved by Hörmander [4] and, in the framework of hyperfunctions, by KawaiKashiwara (see [5]). For the case (b) Kawai has announced (private correspondence) that by extending the theory of Fourier integral operators to the analytic category, he and Kashiwara have proved the result of this note. (Probably under the weaker assumption that $H_{\mathrm{Re} P_{m}}, H_{\mathrm{Im} P_{m}}$ and the cone axis are linearly independent in $Z$.)

The following definition is a slight variation of the definition given in [4] of the analytic wave front set $W F_{a}(u)$ of a distribution $u$.

DEFINITION 1. $\left(x^{0}, \xi^{0}\right) \notin W F_{a}(u)$ if and only if there is a neighborhood $U$

AMS 1970 subject classifications. Primary 35A05, 35A20.

Copyright $(C)$ American Mathematical Society 1972 
of $x^{0}$ and a sequence $u_{N} \in \mathscr{E}^{\prime}$, such that $u_{N}=u$ in $U$ and

$$
\left|\hat{u}_{N}(\xi)\right| \leqq C^{N}(1+|\xi| / N)^{-N},
$$

when $\xi$ belongs to a conic neighborhood of $\xi^{0}$.

The proof of Theorem 1 starts from an alternative characterization of $W F_{a}(u)$ by means of sequences of pseudo-differential operators corresponding to symbols of the following type.

Definition 2. A sequence $\left(a^{N}(x, \xi)\right)$ is said to belong to $\mathscr{A}^{r}$ if for each compact set $K$ there is a constant $C$ such that

$$
\begin{array}{r}
\left|D_{x}^{\alpha} D_{\xi}^{\beta} a^{N}(x, \xi)\right| \leqq C^{N} N^{|\alpha|}(1+|\xi| / N)^{r-|\beta|}, \\
\quad \text { when }|\alpha+\beta| \leqq N / C \text { and } x \in K .
\end{array}
$$

Moreover, we write $\left(a^{N}\right) \sim 0$ if for every compact set $K$ there is a constant $C$ such that

$$
\begin{aligned}
\left|D_{x}^{\alpha} D_{\xi}^{\beta} a^{N}(x, \xi)\right| \leqq C^{N} N^{|\alpha|}(1+|\xi| / N)^{-k-|\beta|}, & \\
& \text { when }|\alpha+\beta|+k \leqq N / C \text { and } x \in K .
\end{aligned}
$$

In the usual way one associates to $\mathscr{A}^{r}$ a space $A^{r}$ of sequences of pseudodifferential operators. The calculus of these sequences of operators parallels the calculus for usual pseudo-differential operators.

REMARK 4. The estimates (1) and (2) are similar to the estimates for the analytic symbols of Boutet de Monvel and Krée [2]. However we have to work with sequences, since we want to include symbols with support in a compactly generated cone.

Definition 3. $\left(x^{0}, \xi^{0}\right)$ is called noncharacteristic with respect to $\left(a^{N}\right) \in \mathscr{A}^{r}$ if and only if there is a closed conic neighborhood $F$ of $\left(x^{0}, \xi^{0}\right)$ and a decomposition $a^{N}=a_{r}^{N}+a_{r-1}^{N}$, with $a_{r-1}^{N} \in \mathscr{A}^{r-1}$, such that

$$
\left|a^{N}(x, \xi)\right| \geqq|\xi|^{r} / C \text { when }(x, \xi) \in F \text { and }|\xi| \geqq C N \text {, }
$$

for some constant $C>0$.

LEMMA 1. $\left(x^{0}, \xi^{0}\right) \notin W F_{a}(u)$ if and only if there is a sequence $\left(a^{N}\right) \in \mathscr{A}^{0}$ such that $\left(x^{0}, \xi^{0}\right)$ is noncharacteristic with respect to $\left(a^{N}\right)$ and

$$
\left|D_{x}^{\alpha} a^{N}(x, D) u(x)\right| \leqq C^{N} N^{|\alpha|}, \quad \text { when }|\alpha| \leqq N / C .
$$

ProOF. We may construct a local parametrix $\left(e^{N}\right)$ such that $\left(e^{N} \circ a^{N}-1\right)$ $\sim 0$ in a conic neighborhood of $\left(x^{0}, \xi^{0}\right)$. Therefore it is sufficient to use symbols of the form $\phi^{N}(x) \psi^{N}(\xi)$, which are equal to one in a conic neighborhood of $\left(x^{0}, \xi^{0}\right)$ when $|\xi| \geqq C N$.

We can now sketch the proof of Theorem 1 . Since the case (a) is very similar to (b) we will assume that (b) is satisfied and afterwards briefly comment on the differences in the case (c). For any sufficiently small conic 
neighborhood $U \times \Gamma$ of a characteristic point $\left(x^{0}, \xi^{0}\right)$ of $P_{m}$ we may, by means of the Cauchy-Kovalevsky theorem, find an analytic symbol $c(x, \xi)$ such that $\left\{c P_{m}, \bar{c} \bar{P}_{m}\right\} \equiv 0$ in $U \times \Gamma$. Assuming now that $\left\{P_{m}, \bar{P}_{m}\right\} \equiv 0$ we can, for an aribitrary small conic neighborhood $F$ of a bicharacteristic strip $b$ in $U \times \Gamma$, construct a sequence $\left(a^{N}\right) \in \mathscr{A}^{0}$ vanishing outside $F$, such that the points on $b$ are noncharacteristic with respect to $\left(a^{N}\right)$ and

$$
\left(\left[P(x, D), a^{N}(x, D)\right]\right) \sim 0, \text { when } x \in U .
$$

The condition $\left\{P_{m}, \bar{P}_{m}\right\} \equiv 0$ means that locally $H_{P_{m}}$ is reduced to $\partial / \partial \bar{z}$ in suitable coordinates. This permits the construction of a sequence $\left(a^{N}\right)$ satisfying the estimates (1) without too much difficulty.

Because of (3) we have

$$
\left|D_{x}^{\alpha} P(x, D) a^{N}(x, D) u(x)\right| \leqq C^{N} N^{|\alpha|} \text {, when }|\alpha| \leqq N / C \text { and } x \in U .
$$

Suppose now that $\left(x^{0}, \xi^{0}\right) \in b \cap \operatorname{compl}\left(W F_{a}(u)\right)$. If $F$ is small enough and coordinates are properly chosen we have, for some $\varepsilon>0$,

$$
\begin{aligned}
\left|D_{x}^{\alpha} a^{N}(x, D) u(x)\right| \leqq & C^{N} N^{|\alpha|}, \quad \text { when }|\alpha| \leqq N / C, \\
& \left(x_{n-1}-x_{n-1}^{0}\right)^{2}+\left(x_{n}-x_{n}^{0}\right)^{2}<\varepsilon \text { and } x \in U .
\end{aligned}
$$

To prove Theorem 1 we just have to prove that under the assumptions (4) and (5) the $\varepsilon$, occurring in (5), can in fact be taken to be $+\infty$.

It is easy to see that by means of a local change of coordinates and a multiplication with an analytic elliptic pseudo-differential operator (see Proposition 6.1 of [4]) this problem may be reduced to the following one.

Let $L(x, \xi)=i \xi_{n}+S\left(x, \xi^{\prime}\right)$ be an analytic symbol (see [2]) in some cone $F \subset X \times R^{n}$. Assume that $L$ is principally normal, i.e., $i\left\{L_{1}, L_{1}\right\}(x, \xi)$ $=\operatorname{Re}\left(L_{1}(x, \xi) \lambda(x, \xi)\right)$ for some $\lambda$ homogeneous of degree zero. Choose a sequence $\left(\chi^{N}\right) \in \mathscr{A}^{0}$ such that $\chi^{N}$ has support in $F$ and equals 1 in the support of $a^{N}$. Put $L^{N}(x, \xi)=L(x, \xi) \chi^{N}(x, \xi)$ and $u_{N}(x)=a^{N}(x, D) u(x)$. Show that if

$$
\left|D_{x}^{\alpha} L^{N} u_{N}\right| \leqq C^{N} N^{|\alpha|}, \quad \text { when }|\alpha| \leqq N / C \text { and } x_{n}<1
$$

and

$$
\left|D_{x}^{\alpha} u_{N}\right| \leqq C^{N} N^{|\alpha|}, \quad \text { when }|\alpha| \leqq N / C \text { and } x_{n}<\min \left(\left|x^{\prime}\right|^{2}, 1\right)
$$

then

$$
\left|D_{x}^{\alpha} u_{N}\right| \leqq C^{N} N^{|\alpha|}, \quad \text { when }|\alpha| \leqq N / C \text { and } x_{n}<\frac{1}{2} .
$$

This may be done by using a variant of a classical inequality of Calderón (see [3]). Let $\Lambda_{N}^{s}$ denote the pseudo-differential operator in $x^{\prime}$-space corresponding to the symbol $\left(1+\left|\xi^{\prime}\right|^{2} / N^{2}\right)^{s / 2}$, and denote by $\|\cdot\|$ the $L^{2}$-norm in $x^{\prime}$-space. Finally put $g_{\sigma}\left(x_{n}\right)=\left(\left(x_{n}-\sigma\right)^{2} / 2-\sigma^{2} / 4\right)$. 
LEMMA 2. Let $L^{N}$ and $u_{N}$ be as above. Then there are positive constants $\sigma, C$ such that

$$
N \int\left\|\Lambda_{N}^{N g_{\sigma}\left(x_{n}\right)} u_{N}\right\|^{2} d x_{n} \leqq C \int\left\|\Lambda_{N}^{N g_{\sigma}\left(x_{n}\right)} L^{N} u_{N}\right\|^{2} d x_{n}+C^{N} \int\|u\|^{2} d x_{n},
$$

when $u \in C_{0}^{\infty}$ and $u_{N}$ vanishes for $\left|x_{n}\right| \geqq \sigma$.

With minor modifications, this lemma may be proved in the same way as Théorème 1 in [6].

REMARK 5. Lemma 2 just gives (8) for derivatives with respect to $x^{\prime}$ but since $L$ is of the form $i \xi_{n}+S\left(x, \xi^{\prime}\right)(6)$ implies (8).

REMARK 6. In the case (c) any sequence $\left(a^{N}\right)=\left(a^{N}(D)\right)$ which is independent of $x$ will trivially satisfy (3). However, since $\left(a^{N}\right)$ does not depend on $x$, (5) will a priori only be satisfied when $\left|x-x^{0}\right|<\varepsilon$. We want to show that, in fact, (5) is satisfied on the whole projection of $b$ onto $U$. Again this will follow from Lemma 2 since we may suppose that the direction of $d_{\xi} P_{m}(\xi)$ in $C^{n}$ is as close as we want to that of $d_{\xi} P_{m}\left(\xi_{0}\right)$ when $\xi$ belongs to the support of $\left(a^{N}\right)$.

REMARK 7. Theorem 1 immediately gives results about global solvability for $P u=f, f \in C^{\infty}$, for any $P$ satisfying (a), (b) or (c) (see [4]).

\section{REFERENCES}

1. K. G. Andersson, Propagation of analyticity of solutions of partial differential equations with constant coefficients, Ark. Mat. 8 (1971), 277-302.

2. L. Boutet de Monvel and P. Krée, Pseudo-differential operators and Gevrey classes, Ann. Inst. Fourier (Grenoble) 17 (1967), fasc. 1, 295-323. MR 37 \# 1760.

3. A. P. Calderón, Uniqueness in the Cauchy problem for partial differential equations, Amer. J. Math. 80 (1958), 16-36. MR 21 \#3675.

4. L. Hörmander, Uniqueness theorems and wave front sets for solutions of linear differential equations with analytic coefficients, Comm. Pure Appl. Math. 24 (1971).

5. T. Kawai, Construction of local elementary solutions for linear partial differential operators with real analytic coefficients. I. The case with real principal symbols, Publ. Res. Inst. Math. Sci. Kyoto (to appear).

6. B. Malgrange, Unicité du problème de Cauchy, d'après A. P. Calderón, Séminaire Bourbaki 1958/59, Exposé 178, fasc. 2, Secrétariat mathématique, Paris, 1959. MR 28 \# 1091. 08903

Department of Mathematics, Rutgers University, New Brunswick, NeW Jersey 